\title{
Turings Maschine und Blacks Box - Mechanische Intelligenz nach dem Feedback
}

\author{
Religious-scale projects - and their comforts - have often scourged humankind. I'm thinking of \\ Egyptian pyramids, Moslem jihads, Mongol hordes, Christian crusades, the Third Reich, world \\ Communism, maybe science itself. Part of their hazard is that they become their own universe - \\ an infinite regress of self-reference grounded nowhere. \\ -Steward Brand, „The Long View“, in: Space Colonies (CoEvolution September 1977). ${ }^{1}$
}

\section{Turing und die blinden Flecken - White Field und Black Box}

,Berechenbarkeit‘ ist - genauso wie ,Einfachheit‘ oder ,Eleganz - ein Erkenntnisideal: Eine Erkenntnis gilt dann als besonders wertvoll, wenn sie aus eindeutigen Elementen zusammengesetzt ist und lückenlos regelgemäß hergeleitet werden kann. Der besondere Wert des ,Berechenbaren' liegt darin, dass wir für das ,Berechnete“ universelle Gültigkeit beanspruchen können - an allen Orten, in jeder Zeit und intersubjektiv für alle rechenbegabten Wesen. Alles was berechenbar ist, ist ,klar ${ }^{`}$ aus ,deutlichen' Elementen zusammengesetzt - es handelt sich um Argumentationsverfahren mit höchster Nachvollziehbarkeit und Transparenz. Eine black box meint das Gegenteil - mindestens einen markierten ,blinden Fleck ${ }^{c}$ im betrachteten System, oft eine bewusst ausgesparte Komplexität, die bloß in ihrer Funktion, nicht aber in ihrem Aufbau erfasst und angezeichnet wird. Alan M. Turing gilt in der Gegenwart als Leittheoretiker der Berechenbarkeit. Was hat ein Beitrag zu Alan Turing nun in einem Band über Black Boxes verloren? Turing, einer der Pioniere elektronischer programmgesteuerter Rechenmaschinen, verwendet den Begriff kein einziges Mal in seinen Schriften. ${ }^{2}$ Außerdem ist er bis in die Gegenwart bekannt als Schöpfer der nach

1 Online: https://space.nss.org/settlement/nasa/CoEvolutionBook (Stand: 19.9.2019).

2 Als aktuelles Referenzmaterial und Textausgaben einschlägig Cooper/van Leeuwen 2013, Cooper/ Copeland 2016 und Copeland et. al. 2017; grundlegende Edition, noch immer unabkömmlich: Die Collected Works, hier vor allem Vol.1: Machine Intelligence von 1992. Online-Recherche in Turings Nachlass im King's College (GB 272 PP/AMT) und in den jüngst digital erschlossenen Neufunden vom Dach-

Dieser Beitrag ist im Heidelberger Sonderforschungsbereich 933 „Materiale Textkulturen. Materialität und Präsenz des Geschriebenen in non-typographischen Gesellschaften“ entstanden (Teilprojekt Ö „Schrifttragende Artefakte in Neuen Medien“). Der SFB 933 wird durch die Deutsche Forschungsgemeinschaft finanziert.

Die Druckfassung entstand im Rahmen des HEiKA - Heidelberg Karlsruhe Strategic Partnership, Heidelberg University, Karlsruhe Institute of Technology (KIT), Germany. 
ihm benannten (Papier-)Maschine ${ }^{3}$, die uns bis heute als universales Modell des Berechnens dient, und zwar im Sinne einer transparenten und eindeutigen Folge von Zeichen, die regelgeleitet operationalisiert werden. Eine Black Box ${ }^{4}$ war (und ist) aber grundsätzlich eine undurchschaubare funktionale Einheit, deren ursprüngliche Aufgabe die Verarbeitung von Rückkopplung und Wechselwirkung war und nicht die lineare Folge. Auch die experimentellen Rechenmaschinen ${ }^{5}$, die er entwarf und mit denen er arbeitete, waren aus diskreten Teilen aufgebaut und zusammengesteckt aus handhabbaren und greifbaren Komponenten. Zu Turings Zeit war die Mechanik des Rechenapparats historisch noch nicht hinter der Oberfläche und dem Interface des Industriedesigns verschwunden und die industrielle Miniaturisierung hatte noch nicht den integrierten Schaltkreis hervorgebracht. ${ }^{6}$ Es scheint also auf den ersten Blick passender, mit Turing ein White Field abzustecken, als über Black Boxes zu schreiben. Diese Einschätzung ist aber aus drei Gründen trügerisch: einem technikhistorischen, einem wissenschaftstheoretischen und einem methodologischen.

\section{Eine Technikgeschichte vor der ,Black Box Computer‘}

Technikhistorisch gab es eine Zeit vor der Versiegelung der ,Black Box Computer ${ }^{7}$ - in diese blicken wir, wenn wir auf Turings Zeit schauen. Das Fallbeispiel ist komplex: Die Black Box, die mit Turing verkoppelt ist, ist das technische Prinzip des Feedback - der prozessualen Rückkopplung - und nicht die Metapher des Verschließens, Versiegelns oder Einboxens. ${ }^{8}$ Der Computer, den Turing $1950^{9}$ in Weiterentwicklung seines Modells von $1936^{10}$ entwirft, arbeitet nicht mehr diskret und nicht mehr linear. Aber wir finden diese ,Turing-Maschine 2.0 $0^{\mathbf{1 1}}$ in seinen Schriften nur, wenn wir diese unkonventionell - eben nicht wie eine Black Box - betrachten, sie ganz genau lesen und in den technischen Kontext ihrer Entstehungszeit stellen. Im Folgenden soll versucht

boden der Universität Manchester (GB 133 TUR/Add.). Der einschlägige Handbucheintrag von Hodges 2013 [2002] („Alan Turing“, SEP) liefert eine gute Auswahl von Zitationen und Anküpfungsknoten.

3 Das Konzept der ,Papiermaschine‘ greift ausführlich Dotzler 2005 auf.

$4 \mathrm{Zu}$ Begriff und Geschichte der Black Box siehe die Einleitung dieses Bandes von Geitz/Vater/Zimmer. 5 Zur Geschichte der (elektronischen) Rechenmaschinen Hartree 1949, de Beauclair 1968, Goldstine 1972, Metropolis/Howlet/Rota 1980, Williams 1985. Zu Turings Maschinen in Manchester Lavington 1975.

6 Zu einigen Aspekten des Computersystem-Designs siehe Arnold in diesem Band.

$7 \mathrm{Zu}$ einer ausführlichen Darstellung aus der Perspektive der kontinentalen Theorie siehe die gleichnamige Dissertation von Becker 2012.

$8 \mathrm{Zu}$ den verschiedenen Typen der Black Box siehe Geitz/Vater/Zimmer in diesem Band.

9 Turing 1950.

10 Turing 1936.

11 Oder ,Feedback-Turing-Maschine', hier fehlt eine eingeführte Bezeichnung (f-type-machine). Die Vermutung, es könne sich hierbei um das (oder ein Element des) Oracle handeln, kann hier nur geäußert, aber nicht verfolgt werden. 
werden, dunkle Stellen in Turings klassischem Text aus der Kenntnis seiner Praktiken und vor dem Bühnenbild ${ }^{12}$ seiner Artefakte zu verstehen. Diese dunklen Textstellen sollen nichts Geringeres als die Entkräftung des „Arguments der Lady Lovelace“ und die Grundlegung des „Maschinellen Lernens“ leisten: (1) Eine Maschine könne nichts Neues hervorbringen, sondern befolge immer nur die Regeln, die wir ihr vorgegeben haben - so der ,Einwand der Lady Lovelace‘. ${ }^{13}$ (2) Da eine Maschine durch ihren Bauund Schaltplan determiniert sei, könne sie nicht lernen. ${ }^{14}$ Turings Maschinen sollen nun aber beides leisten: sie sollen Non-Lovelace-Maschinen sein (die ein ,Lovelace-Limit‘ überschreiten und somit Neues hervorbringen könnten). Eine Eigenschaft, die ihnen diese Fähigkeit sichern würde, wäre, dass sie ,lernen` können - ähnlich wie menschliche Kinder im Schulunterricht. ${ }^{15}$ Turing benötigte also in der Theorie ein neues Maschinen-Konzept und für die Praxis neue Bauteile. Ein neues Maschinen-Konzept fand er in der Kybernetik: diese griff auf elektromagnetische Bauteile zurück, die das Verschalten von Rückkopplung mit dem Ziel der Selbststeuerung ermöglichten.

Da in der Gegenwart - und in diesem Band - sowohl die eingekapselte Hardware der Rechenmaschinen als auch die verschlüsselte Software, die sich in diesen verkörpert, als Black Box wahrgenommen, behandelt oder analysiert werden, ist es umso bezeichnender, dass Turing seine Computer unter Rückgriff auf konkrete Black BoxTechnologie der Früh-Kybernetik weiterentwickelte - so wird aus den historischen Artefakten in gewissem Sinne herausgelesen, was in sie im Entwurf eingeschrieben wurde.

\section{Gibt es ein Computingdispositiv? - Zwiebelschalen und Bausteine}

Wenn ich den Anlass habe, eine wissenschaftshistorisch ,blinde Stelle‘ zu vermuten - oder metaphorisch eine ,Black Box‘ öffnen will -, benötige ich eine Methode. Die rezent vorherrschende ,Logische Analyse taugt nur begrenzt für historische Untersuchungen und wird typischerweise ahistorisch verwendet. Umso dringlicher ist der Blick auf vorhandene Alternativen.

12 Für einen theaterwissenschaftlichen Blick auf den Computer Laurel 1991.

13 „The Analytical Engine has no pretensions to originate anything. It can do whatever we know how to order it to perform“ zitiert Turing Lovelaces Memorandum über Babbages Rechenmaschine (Kursivierung bei Turing nach Lovelace, Turing 1950, 450).

14 Ausführlich Turing 1950, $454 \mathrm{ff}$.

15 Turing 1950, 455ff. Beispielhaft zur child machine: „We normally associate punishments and rewards with the teaching process. Some simple child-machines can be constructed or programmed on this sort of principle.“, Turing 1950, 457. 
Auch wenn Michel Foucault explizit kein Arbeitsprogramm in Form einer Verfahrensbeschreibung für ,Diskursanalysen“ oder ,Wissensarchäologie“ vorgelegt hat, können wir uns doch an einer flüchtigen offenen Skizze orientieren, die er 1977 in einem Interview mit dem deutschen Titel „Das Spiel des Michel Foucault“ entwirft. ${ }^{16}$ Das dort skizzierte Vorgehen kann im vorliegenden Beitrag nur bausteinhaft durchgeführt werden. Blicke auf (a) Turings Praktiken des ,Rechnens“ und des ,Programmierens' als ,Genanntes‘, (b) die strategische Funktion der Rechenmaschinen in Turings Zeit und (c) auf Turings Einbettung in Kommunikationsnetzwerke und Forschungsgruppen ermöglichen es uns, ein (d) ,Ungenanntes‘ aufzuspüren, das sich zwischen den Maschen des Netzes des Computingdispositivs ${ }^{17}$ zeigt. Dieser Fund kann als Einzelfall vermitteln, was es bedeutet, wenn die logische Analyse um eine Darstellung der epistemischen Praktiken, der am Erkenntnisprozess beteiligten Artefakte und die Kommunikations-Netze im sozialen Raum erweitert wird - kurz, wenn ich Kulturtechniken und Technikkultur berücksichtige. ${ }^{18}$

\section{4 - Der Computer - Die Leittechnologie der Turing-Galaxis}

Der Computer ist eine Technologie der symbolischen Repräsentation und der Kommunikation, kurz - eine Technologie des Schreibens. ${ }^{19}$

Computer sollen Schreibmaschinen sein - keine elektronischen Rechenknechte. Diese apodiktische Einschätzung stellt der Beforscher des Medienwandels David J. Bolter gegen eine geläufige Haltung, die unsere programmierbaren elektronischen Rechenapparate nicht als ,Werkzeuge“ beschreibt, sondern als ,Wesen“ zu fassen versucht - nicht als Erweiterung des Apparates menschlicher Fähigkeiten, sondern als potentieller Träger einer nicht ,geborenen‘, sondern ,künstlichen‘ Intelligenz. Bolter kennt sich mit der Rolle von Technologie als Ausdruck und Voraussetzung menschlicher und kollektiver Kognition aus - schon seine Kulturgeschichte des Schreibund Rechengeräts von 1984 ist ein Versuch, die Einbettung des Menschen der westlichen Welt in eine dominant technologische Kultur zu erkunden. Im symbolisch

16 Foucault 2003 [1977], 292-293.

17 „Computingdispositiv“ als Dispositiv-des-Rechnens hat kürzlich Moritz Hiller in seiner Einleitung zur Sektion „Computing“ des Weimarer Readers Grundlagentexte der Medienkultur (Hiller 2019) eingeführt. An dieser Stelle soll versucht werden, ihn operabel zu machen, um seine Brauchbarkeit als Erkenntnisinstrument zu erproben.

18 Nicht uninteressant ist hierbei eine gewisse Rekursivität des Gedankengangs - die Untersuchungen verknoten sich sogar zu einer seltsamen Schleife.

19 Bolter 1987. 
aufgeladenen Jahr 1984 hat er das Ziel, Turing's Man $^{20}$ seiner unmittelbaren Gegenwart besser verstehen zu lernen. Jede Kulturepoche habe ihre Leittechnologie, so Bolter, und diese ,Leittechnologien“ seien eben deshalb bedeutsam, weil sie nicht nur den manuellen Werkzeuggebrauch prägten, sondern auch als Metapher kognitiven Gewohnheiten und Praktiken Gestalt geben würden. Der ,Turing-Mensch“ sei konfrontiert mit einer Leittechnologie neuen Typs: einer universellen und vernetzten Medienmaschine - dem durch das Internet verbundenen persönlichen Digitalcomputer. Diesen Gedankengang fasst Bolter im selben Jahr in Daedalus prägnant zusammen - die Leittechnologie wird zur Leitmetapher:

We do not have to become religious converts to artificial intelligence in order to appreciate the computer metaphor. Nor do we have to join in the sterile debate over the question of whether computers can really think. Instead, we can ask in what ways the metaphor is apt and in what ways it may fail. Our view of the human mind changes from age to age, based upon social conventions, the work of poets and philosophers, and the current technical metaphors. Surely our contemporary task is to come to terms with the new electronic technology, a task that permits neither a complete rejection nor blind acceptance of the computer metaphor. ${ }^{21}$

Aus dieser Sicht wird ,Künstliche Intelligenz‘ zu einem in der Sache überschätzen, aber erklärbaren und spannenden Phänomen: Genauso, wie bereits andere historische Technologien als Wunderwerke der Erfindungskraft die epistemische Phantasie der Wissenschaftler`innen einer Zeit anregten, ergeht es uns heute mit dem programmierbaren vernetzten Digitalcomputer. Er wird damit $\mathrm{zu}$ einer bedeutenden Metapher. ${ }^{22}$ Als Sprachspiel im brisanten Feld epistemischer Selbstbespiegelung und Selbstzuschreibung leitet die Computermetapher Praktiken des Wissens, ähnlich wie einst das Uhrwerk (oder davor das Pendel) als Bildgeber, Vorbilder und Modelle des Geistes dienten. ${ }^{23}$

Wolfgang Coy vollzieht im selben (symbolgeladenen) Jahr in Enzensbergers Kursbuch zur „Computerkultur“ ebenfalls die Wechselwirkung zwischen wissenschaftlicher Modellbildung und Leittechnologie nach:

Aus dem Hebelmodell der Renaissance, sichtbar in Giovanni Battista Bracellis eigenartigen Graphiken des Maschinenmenschen und perfekt dargestellt in Leonardo da Vincis anatomischen Skizzen [...], über das Pumpen- und Kreislaufmodell im Gefolge der thermodynamischen Entdeckungen, entstand das Steuermodell der Nervenstränge in der Kybernetik und das moderne Paradigma des Menschen als eines informationsverarbeitenden Systems [...]. ${ }^{24}$

20 Bolter 1984a.

21 Bolter 1984b, 17.

22 Zur Entstehung von Metaphern und möglichen Rollen im Erkenntnisprozess siehe Schütt in diesem Band.

23 Zur Welt-als-Maschine in der Neuzeit siehe McLaughlin 1994.

24 Coy 1984, 2. 
Heute sind es allerdings nicht mehr die mechanischen Flötenspieler und UhrwerkEnten ${ }^{25}$, die uns als Theatermaschinen des federgetriebenen Welttheaters entgegentreten, es sind ELIZA, A.L.I.C.E. oder EUGENE GOOSTMAN, die aus der interfacevermittelten Bootstrap als leuchtende Avatare ${ }^{26}$ auf dem Bildschirm emporsteigen.

\section{Die Reduktion auf logische Analyse verfehlt die Hälfte}

Diese kultur- und technikhistorischen Überlegungen werden jedoch nicht allgemein geteilt. Es gibt einen analytischen Impetus, der geisteswissenschaftliche Forschung genauso auf logische Strukturen reduzieren möchte, ${ }^{27}$ wie den Geist auf die Strukturen des Materials, über dem er ablaufe. ${ }^{28}$

Wissen wird jedoch nicht gefunden, es wird gemacht: ${ }^{29}$ in einem Verfahren, das ganz zutreffend ,Wissenschaft' heißt. An diesem Verfahren der Herstellung von Wissen sind Wissenschaftler`innen beteiligt, deren Gruppen sozial verfasst sind, und die an speziell für die Wissensherstellung hergerichteten Orten mit zu diesem Zweck geformten Werkzeugen arbeiten. Um zu verstehen, wie Wissen entsteht, wird es helfen, eine Topographie der Wissensorte zu kartieren, die Praktiken der Wissensherstellung $\mathrm{zu}$ erfassen und die Gestalt der Artefakte zu beschreiben, anhand derer diese Wissenspraktiken vollzogen werden und wurden.

Soll die Spur der Genese der Episteme aufgenommen werden, ist also historisches Arbeiten erforderlich. Hierfür reicht eine logische Analyse gegebener Texte nicht aus das Erkenntnisinteresse ist nicht erschöpft, wenn der propositionale Gehalt erfasst und ein Argument isoliert ist. Die Reduktion eines Textes auf seine ,logische Struktur

25 Einen guten Überblick über clockwork automata liefert Riskin 2016.

26 In historischer Reihenfolge die Benennungen der einschlägigen und archetypischen ChatBots von Joseph Weizenbaum (1966), Richard Wallace (1995) und WladimirWeselow u. Eugene Demchenko (2001).

$27 \mathrm{Zu}$ den Schwierigkeiten und Herausforderungen dieses Ansatzes bereits - ambivalent - Quine 1951, 31: „Analyticity at first seemed most naturally definable by appeal to a realm of meanings. On refinement, the appeal to meanings gave way to an appeal to synonymy or definition. But definition turned out to be a will-o'-the-wisp, and synonymy turned out to be best understood only by dint of a prior appeal to analyticity itself. So we are back at the problem of analyticity." Der im vorliegenden Beitrag vorgeführte und erprobte Ansatz geht hingegen davon aus, dass ,Bedeutung“ im Prozess der Wissenspraktiken ausgelesen, gespeichert, operationalisiert und übertragen wird. Werkzeuge - oder ,Denk-Zeuge‘ - die dieser Unternehmung dienen, sind zum Beispiel ,Netzwerk', ,Entfaltung‘, ,Kartierung، oder andere Techniken einer ,synthetischen' Philosophie.

28 Eine bemerkenswerte Zuspitzung der materialistisch-naturwissenschaftlichen Reduktion (sozusagen für ,ganz Helle') liefert entlang seiner Leitmetapher der survival machine streitbar Dawkins 2006 [1976], passim, aber beispielhaft 49: „The main way in which brains actually contribute to the success of survival machines is by controlling and coordinating the contractions of muscles." Vgl. in diesem Band Maschewski/Nosthoff.

29 Auch mit Latour, z. B. 1987. 
bleibt bei einem genetischen Erkenntnisinteresse genauso unbefriedigend, wie materialistisch reduktionistische Modelle des Menschen, wenn es um ,Kunst“, ,Wahnsinn“ oder ,Glück' geht. Werden sehr große, sehr komplexe und sehr alte Systeme wie die menschliche Kultur, in der Phänomene wie ,Wissen' oder ,Technik ${ }^{\star}$ - auch in ihrer Entstehung und Umbildung - erst möglich werden, untersucht, muss der Werkzeugkasten vergrößert und der Untersuchungsgegenstand schrittweise (oder schichtweise) erweitert werden. Sind die Felder irgendwann so groß, dass über sie nach einer Analyse der logischen - also der sprachlich-symbolischen - Form nichts mehr gesagt werden kann und daher geschwiegen werden müsste, entsteht der Wunsch nach einem anderen Gerät der Feld-Vermessung. Genau dieses Gerät der Erweiterung des ausmessbaren Feldes wissenschaftlicher Erkenntnis wurde im vergangenen Jahrhundert nach der radikalen Selbstbegrenzung auf ,das Argument“ wieder vergrößert: erst durch Sprachspiele, dann durch die Untersuchung von Praktiken der Wissensherstellung, auch der Rolle der beteiligten (Labor-)Artefakte, schließlich durch den Blick auf die kulturelle Verfasstheit der Bedingungen der Entwicklung, Geltung und Vermessung unserer Ideen und Konzepte selbst. Diesen letzten Erweiterungsschritt können wir vorläufig einen Schritt in die ,Episteme‘ nennen.

Interessieren Bolters kulturelle und kulturgestaltende Metaphern, zu denen die Leittechnologie einer Zeit kognitiv gerinnt, so ist die logische Analyse jedenfalls nur begrenzt aussagekräftig, da dort Künstliche Intelligenz nur im Text stattfindet und zwar in wenigen ausgewählten Kerntexten einer klar abgrenzbar gedachten Debatte. Die Anweisungen sind hier: Finde die - wenigen - zentralen Texte; finde die dort vorgebrachten Argumente und zeige ihre Argumentationsstruktur. Kurz: Filtere das Unwesentliche aus dem Text, die Störungen und das Geräusch, die Artefakte der Rede und des Stils, und bewahre und verstärke das Wesentliche - die logische Struktur und den propositionalen Gehalt. Dieses reduktionistische Programm der logischen Analyse lässt dabei nicht viel von einem Text übrig und noch viel weniger vom Reichtum der historischen Phänomene. ${ }^{30}$

Wird Turing in der Praxis der Logischen Analyse gelesen, werden seine Texte auf ein Argument reduziert - vielleicht illustriert durch ein didaktisierbares Gedankenexperiment $^{31}$, das Imitationsspiel. Eine belastbare Fassung dieser logischen Struktur liefert Steward Shieber ${ }^{32}$ als ,Turing Syllogismus ${ }^{633}$ :

30 Der Vorteil ist, dass es eine Methode liefert, die außerordentlich sichere und vergleichbare Ergebnisse liefert, die als Handwerk lehr- und lernbar sind.

31 Die Beschäftigung mit Gedankenexperimenten kann als Hilfsoperation aus Mangel an empirischem Material gedeutet werden: Können keine Artefakte oder Praktiken untersucht werden, muss man sich eine Geschichte ausdenken, um überhaupt über irgendeinen Stoff reden zu können (Dank an Peter McLaughlin für diese Spur).

32 Shieber 2004, 136.

33 Wobei vier (!) Prämissen unelegant sind, da es sich anscheinend um einen sehr voraussetzungsreichen Schluss handelt. 


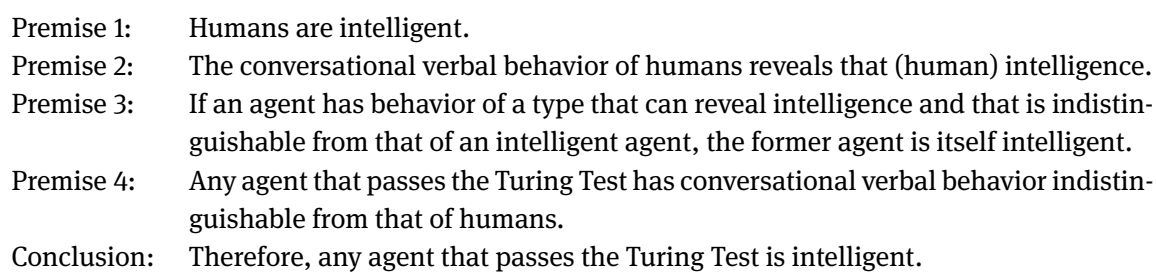

Turings Beitrag zur Geschichte des Nachdenkens über Menschen und Maschinen wird so eingefaltet zu einem handlichen Datenpaket, das im Fachdiskurs operationalisierbar ist. ${ }^{34} \mathrm{Er}$ selbst macht sich jedoch in einem Brief an seinen Freund Norman Routledge 1952 über die Knappheit von Syllogismen (bittersüß) lustig:

Glad you enjoyed broadcast. J[efferson] certainly was rather disappointing though. I'm rather afraid that the following syllogism may be used by some in the future

Turing believes machines think

Turing lies with men

Therefore machines do not think

Yours in distress, Alan.

Es ist durchaus berechtigt, auch Fragen nach der Entstehung der Geltung von Turings Arbeit zu stellen, nach den historischen Bedingungen seiner Wirksamkeit. Sie ist ein Produkt von Auswahlprozessen, Verdichtungen und Verarbeitungen - der Prozessierung von Sachverhalten und Tatsachen, guten Einfällen und Phantasien.

\section{0 - Bausteine der Turing-Zeit}

Der erste Band der Schriften Alan M. Turings, den David Ince 1992 veröffentlichte, trägt den Titel Mechanical Intelligence. Unter diesem Titel sammelte der Herausgeber die Aufsätze und Redemanuskripte Turings, in denen er sich mit automatischen Rechenmaschinen und ihrer Programmierung beschäftigt - es geht im Detail um Spiel-Programme wie Schach oder Dame, aber auch generell um lösbare oder unlösbare Probleme. Er enthält außerdem einen inzwischen klassisch gewordenen Aufsatz der frühen Informatik, der aufgrund seines Themas und seines zugänglichen und unterhaltsamen Stils heute interdisziplinär gelesen wird: Computing Machinery and Intelligence ${ }^{35}$ von 1950. Zuerst veröffentlicht im britischen MIND-Magazin wird er

34 Hiermit könnte man zufrieden sein und sich nach geprüfter Geltung im Raum alternativer Strukturen um paradigmatische Konkurrenten bemühen, die jedoch nicht empiriegesättigt sein müssten. Die Suche nach Fallbeispielen und Anwendungsfällen, Artefakten und Praktiken ist ein anderer Weg. 35 Als editionskritische Ausgabe mit Konkordanz zum Original siehe Shieber 2004. Als korrigierte deutschsprachige Übersetzung Kittler/Dotzler 1987. Als kommentierte Ausgabe (mit Laufkommentar 
zum zentralen Bezugspunkt der KI-Forschung und ihrer Diskussion bis in die Gegenwart. Zu seinem zentralen Gedankenexperiment - dem ,Imitationsspiel‘ - und zur Turing-Maschine als Kernkonzept wurden gehaltvolle Beiträge vorgelegt - sowohl was logische Struktur als auch historische Einbettung angeht. ${ }^{36}$ Im Folgenden soll versucht werden, den historischen Geltungsbedingungen näher zu kommen.

\section{Das Genannte - Turings Praktiken ,Rechnen“ und ,Programmieren“}

Die moderne Geschichte der KI beginnt mit dem grandiosen Scheitern des Forschungsprogramms universeller Berechenbarkeit. Turings Motivation, eine „Denkmaschine“ zu bauen, war ursprünglich, einen Angriff auf eine der größten Hoffnungen der Mathematiker abzuwehren: Kurt Gödel meinte, bewiesen zu haben, dass - sehr vereinfacht ausgedrückt - die ,Weltformel‘ oder ,das System von (wirklich) Allem' nicht herstellbar sei. Turing trat den Gegenbeweis an und konstruierte einen Automaten, der beweisen sollte, dass Gödel unrecht hatte. Dieser Beweisautomat sollte unangreifbar arbeiten, seine Ergebnisse sollten intersubjektiv zustimmungspflichtig sein: Schritt-für-Schritt - also ohne intuitive Sprünge - und mit vollständig bekannten operationalen Regeln sollte vollständig nachvollziehbar und deterministisch der Beweis erbracht werden. Aber: Turings Versuch ging fehl; er konstruierte zwar das gültige Modell der Berechenbarkeit - das wir bis heute ,Universelle Turing Maschine' nennen -, doch diese Turing-Maschine lieferte ihm ärgerlicherweise den Beweis, dass Gödel recht hatte und zwar unangreifbar und intersubjektiv zustimmungspflichtig. Jeder, der ein wenig Zeit und Willen mitbringt, kann diesen Beweis nachvollziehen: Ausgerüstet mit Stift, Radiergummi, einem passenden Rechen-Regelbuch und einer ausreichend großen Menge Papier kann sich jeder rechnende Mensch in diese Turing-Maschine verwandeln. Um Turing hier kurz und narrativ zu fassen: ${ }^{37}$ Jedes Programm, das eine universelle Rechenmaschine in eine spezielle Problemlösungsmaschine verwandelt, muss in einer formalen, ,technischen' Sprache gefasst sein. In jeder dieser Sprachen ist es nun aber möglich, Widersprüche zu produzieren, die dem klassischen Lügnerparadox gleichen. ${ }^{38}$ Ursache hierfür ist die Möglichkeit, selbstreferentielle Arbeitsanweisungen in die Maschine einzugeben. ${ }^{39}$ Sind hier keine

\footnotetext{
von drei Parteien - Hayes et. al.; Saygin; Harnard - und Endkommentar eines vierten Forschers Lucas) Epstein 2000. Zu aktuellen Forschungsfragen und -ansätze Cooper/Van Leeuwen 2013.

36 Zum Imitationsspiel siehe insbesondere Shieber 2004, zur Turing-Maschine insbesondere Herken 1988 und Davis 2000.

37 Ausführlich bei Herken 1988 und Davis 2000.

38 In etwa: „Alle Kreter lügen, sagt Karl der Kreter“. Oder auch: Die Behauptung in Fußnote 38, Satz 2 ist falsch.

39 In etwa dieser Form - die Benennung der Zeile in eckigen Klammern ist ihre ,Cantor-Nummer: [Zeile 1] » Schau Dir Zeile 1 an.
} 
Abbruchbedingungen vorgegeben oder werden diese nicht erreicht, verfängt sich die programmierte Rechenmaschine in ,Endlosschleifen ${ }^{640}$ und arbeitet weiter, ohne abzubrechen. ${ }^{41}$ Tragischerweise ist dieses Verhalten nicht vorhersehbar - das Verhalten einer Turing-Maschine kann nicht von einer anderen Turing-Maschine ,abgekürzt ${ }^{\star}$ prognostiziert werden. Sie muss zu Ende rechnen und hört damit irgendwann auf oder eben nicht.

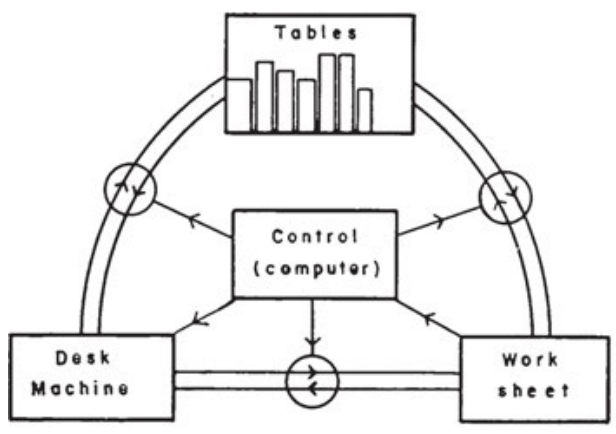

Fig. 39. Organisation of a hand computation.

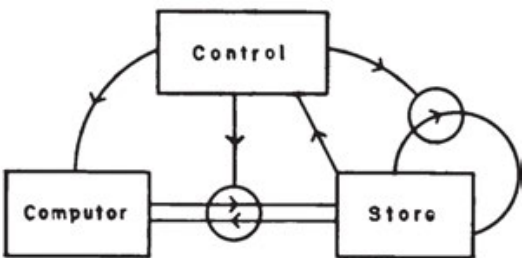

Fig. 40. Organisation of an automatic calculating machine.

Abb.1: 1949: Hartrees Diagramme der Rechenoperationen - von Hand und automatisiert. In von Neumanns und Turings grafischer Notation, die auf McCullogh u. Pitts Notationsvorschlag für das mathematische Modell der Nervenzelle aufbaut.

Prägend waren allerdings nicht nur der Begriff und das Konzept der Turing-Maschine, auch Turings Vorschlag, was Programmieren sei, wurde richtungsweisend: Der erste Schritt ist die Beobachtung von Menschen, die eine Aufgabe erledigen - zum Beispiel als Angestellte in einem Rechenraum arbeitsteilig und getaktet Zinseszinsrechnungen durchführen oder als Mathematiker das Gödelsche Entscheidungsproblem durchrechnen. Dann wird das beobachtete menschliche Verhalten in kleinstmögliche Einheiten zerlegt und ein Modell konstruiert. Dieses Modell wird dann in eine passende formale Sprache, mit der das Verhalten einer Rechenmaschine steuerbar ist, überführt. Die wirklich verblüffende und überraschende Erkenntnis Turings im Scheitern: diese Maschine kann tatsächlich konstruiert werden, aus mechanischen und - noch effektiver - aus elektronischen Einzelteilen. Turing-kompatible

40 Umgangssprachlich: „hängt sich auf“, ein Zeichen: der bluescreen-of-death.

41 Zur Frage der ,Abbruchbedingung‘: Turing sieht ursprünglich vor, dass auch seine ,zirkelfreien“ „automatic machines“ (a-type) nicht aufhören zu arbeiten (siehe Turing 1936) - dies ist so gewollt und kein Fehler. Diese Darstellung ist - vor allem aus Sicht der heutigen Grundlagenvorlesungen der Informatik - interessant und soll an anderer Stelle diskutiert und hier nur markiert werden (Dank an Fabian Dequis für Hinweis und Diskussion). 
programmierbare Rechenmaschinen wurden möglich. Sie wurden gemäß einer ,von Neumann-Architektur` während des Zweiten Weltkriegs physisch konstruiert und so mechanisch ,verkörpert‘.

\section{Die strategische Funktion - Turings Maschinen und das Nachrichtenwesen im ,Heißen' und ,Kalten' Krieg}

Zwei Voraussetzungen für das Gelingen eines Forschungsprojektes sind seine Versorgung und Organisation - dies gilt ganz besonders für Vorhaben, von denen bei Projektstart bekannt ist, dass sie historisch bereits spektakulär gescheitert sind. ${ }^{42}$ Diese logistischen Voraussetzungen sind strategisch und werden im politischen Raum geschaffen; sie sind zunächst unabhängig von der Belastbarkeit der bearbeiteten Ideen und der Forschungsmethoden selbst. Einen elektronischen automatischen Großrechner zu bauen, ist eine aufwendige Angelegenheit, vor allem, wenn es um Prototypen geht. Nicht nur, dass teure und seltene Bauteile zusammengesammelt und passgenau eingefügt werden müssen, man benötigt dafür auch geeignetes Personal im Entwurf, in der Umsetzung und in der Bedienung. Wenn nun ein Bauprojekt in Konkurrenz zu anderen Bauprojekten mit ähnlicher Dringlichkeit steht, werden notwendig Entscheidungen der Ressourcenverteilung gefällt. Dies gilt besonders in Krisensituationen wie einem Weltkrieg. Höchstwertige Radioröhren werden auch für die Radarund Sonarentwicklung benötigt. Hollerith-Lochkarten-Apparate werden auch für die Unterstützung und Beschleunigung manuell organisierter Rechenoperationen eingesetzt. ${ }^{43}$ Für Fachpersonal, das Probleme modellieren, Algorithmen entwickeln und auf hohem Niveau rechnen kann, herrscht ein genereller Bedarf. Ein Projekt zum Bau eines prototypischen Großrechners steht also in Konkurrenz und muss politisch priorisiert werden, um erfolgreich zu sein. Vorgängerprojekte - wie das von Charles Babbage in der ersten Hälfte des 19. Jahrhunderts - scheiterten nicht nur am schlechten Projektmanagement, sondern auch an mangelnder Priorisierung der ressourcenzuweisenden Institutionen. ${ }^{44}$ Im Gegensatz zu Konrad Zuse, dessen Arbeit nicht strategisch gefördert wurde, ${ }^{45}$ oder Gispert Hasenjäger, dessen Arbeitsbedingungen in der deutschen Kryptologieforschung kafkaesk anmuten, ${ }^{46}$ wurden Alan Turing und seine

42 In diesem Fall nennenswert sind Leibniz und Babbage, ein Überblick findet sich bei Pratt 1987.

43 Für eine Detailbeschreibung lochkartengestützter Rechenprozesse siehe Feynman 1997, Kap. „Los Alamos from Below“.

44 Siehe die ausführlichen Klagen in Babbages Schriften, vor allem Babbage 1864, Kap. 6.

45 Siehe die ebenfalls ausführlichen Klagen Zuses in dessen (ebenfalls menschelnder) Autobiographie, insbesondere Zuse 2007 [1970], Kap. 4.

46 Knapp zusammengefasst in Schmeh 2009 und Schmeh 2013. Hasenjäger wurde nach dem Krieg Assistent des Logikers Heinrich Scholz in Münster (der als erster Professor für mathematische Logik und Grundlagenforschung schon in der Zwischenkriegszeit mit Turing korrespondierte) und später Professor in Bochum. 
Kollegen der Government Code and Cypher School in Bletchley Park von Anfang an bestens finanziert und versorgt. Vergleichen wir zwei Berichte und beginnen bei Zuse:

Das Gerät Z2 stand bei meiner Einberufung kurz vor der Vollendung. Dr. Pannke richtete an meine Dienststelle ein Urlaubsgesuch mit der Begründung, daß ich meine Arbeiten ordnungsgemäß übergeben solle. Er schrieb sinngemäß, ich arbeitete an einer großen wissenschaftlichen Rechenmaschine, die auch im Flugzeugbau verwendet werden könne. Dieses Schreiben übergab ich meinem Hauptmann, der es sogleich weiterleitete. Der Bataillonskommandeur, ein Major, bestellte mich zu sich, eröffnete mir zunächst, daß ich als ganz junger Soldat ohnehin kein Recht auf Urlaub hätte, und fuhr fort: „Was heißt hier, Ihre Maschine kann im Flugzeugbau verwendet werden? Die deutsche Luftwaffe ist tadellos, was braucht da noch berechnet zu werden?“ - Was hätte ich darauf erwidern sollen? Der Urlaub wurde nicht gewährt. ${ }^{47}$

Dieser Unwille von offizieller reichsdeutscher Seite, Ressourcen in Belange des wissenschaftlichen Rechnens, insbesondere der Kryptographie und Kryptoanalyse zu lenken, zeigte sich auch deutlich daran, dass in der für die technische Sicherheit der Kommunikationskanäle zuständigen Abteilung des deutschen Generalstabs nur vier Mathematiker ohne Hilfskräfte arbeiteten, mit erwartbaren Folgen:

The story of Gisbert Hasenjaeger renders another possible explanation for the German failure: The Germans did not put enough effort in challenging their own cipher machines. The discrepancy is obvious: whereas at OKW-Chi one inexperienced cryptologist cared about potential Enigma weaknesses, the British put forth their best mathematicians, including Alan Turing and Dilly Knox. A workforce of several thousand people at Bletchley Park supported them..$^{48}$

Wie bereits dieser Vergleich zeigt, sahen die Arbeitsbedingungen Alan Turings gänzlich anders aus: Er arbeitete in einer Regierungskultur, in der Kryptologie geschätzt und gefördert wurde, was sich auch in der Einrichtung eines zentralen Ausbildungsund Forschungszentrums (Bletchley Park) ${ }^{49}$ sowie in den Priorisierungsgewohnheiten des britischen Premierministers zeigte. Nach der Niederlage Frankreichs auf dem Kontinent und vor dem offiziellen Kriegseintritt der USA drohte Großbritannien für einige brisante Monate vom Überseenachschub abgeschnitten $\mathrm{zu}$ werden. Reichsdeutsche U-Boote versenkten schneller Transportschiffe als nachgebaut werden konnte. Grund hierfür war die Güte der elektro-mechanischen Verschlüsselung des koordinierenden Funkverkehrs - ENIGMA konnte nicht schnell genug in hinreichendem Umfang mitgelesen werden. In dieser Situation wies der Kriegs-Politiker Winston Churchill nach beeindruckenden Vorerfolgen der Gruppe um Turing und eingängiger Einforderung verbesserter Ressourcenzuteilung durch die Fachwissenschaftler per Tagesorder am 22. Oktober 1941 seinen Regierungsapparat an:

47 Zuse 2007 [1970], 50.

48 Schmeh 2013, 433.

49 Für einen ersten Eindruck siehe Hodges 1983. 
ACTION THIS DAY

Make sure they have all they want on extreme priority and report to me that this has been done. ${ }^{50}$

Wie Friedrich Kittler und Bernhard Dotzler in ihrem Nachwort zur deutschen Ausgabe der Schriften Turings 1987 zusammenfassen: Turings Maschinen, „die Automatisierung der Mathematik selber“ ${ }^{51}$ entschieden einen Krieg, dieser wurde technologisch geführt und neben der Atombombe waren die Apparaturen der Nachrichtentechnik dafür zentral.

All dies kann Turing 1950 unter dem Official Secrets Act nicht veröffentlichen auch nach Ende des ,Heißen` Weltkriegs besteht nicht nur Churchill auf Wahrung der Geheimnisse, um den strategischen, technologischen Vorteil im ,Kalten' Weltanschauungskrieg nicht zu verlieren..$^{52}$ So kann Turing 1950 nur knapp versichern:

The reader must accept it as a fact that digital Computers can be constructed, and indeed have been constructed, according to the principles we have described, and that they can in fact mimic the actions of a human Computer very closely. ${ }^{53}$

Oder noch kürzer:

There are already a number of digital Computers in working order. ${ }^{54}$

\section{Briefnetzwerke und Forschergruppen - Turings diskursive Verbindungen}

Turing wird in der gegenwärtigen Pop-Kultur zu Unrecht als Sonderling und Eigenbrötler dargestellt. Das Leinwandbild täuscht: Zwar stellt Benedict Cumberbatch ${ }^{55}$ 2014 in The Imitation Game einen Turing dar, der als vereinzelter Freak mit AspergerSymptomatik sein Elektronenhirn alleine in einer abgelegenen Scheune zusammenlötete, doch das biographische Vorbild war gut eingebettet in wissenschaftliche Netzwerke und Clubs. Er entwickelte als Mitglied der Royal Society ${ }^{56}$ den Colossus, die ACE und die „Software“ des MARK I in Manchester. Zwei gute Beispiele für Turings Integration in internationale Forschungsnetzwerke liefert seine Korrespondenz.

50 Abgedruckt in Hodges 1983, 279.

51 Kittler/Dotzler 1987, 213.

52 In seiner stark biographisch geprägten Geschichte des Zweiten Weltkriegs stellt Churchill entweder überlegene britische Spione oder aber bessere Radar-Technologie als kriegsentscheidend dar - auf der Anspielungsebene aufschlussreich ist allerdings Bd. 2, Kap. 19 „The Wizard's War“ (Churchill 1950).

53 Turing 1950, 438.

54 Turing 1950, 436.

55 Bei aller Begeisterung für Cumberbatchs treffliche Holmes-Verkörperung.

56 Gewähltes Mitglied 1951, vorher Grants und Partizipation an Förderung über Stellen. 


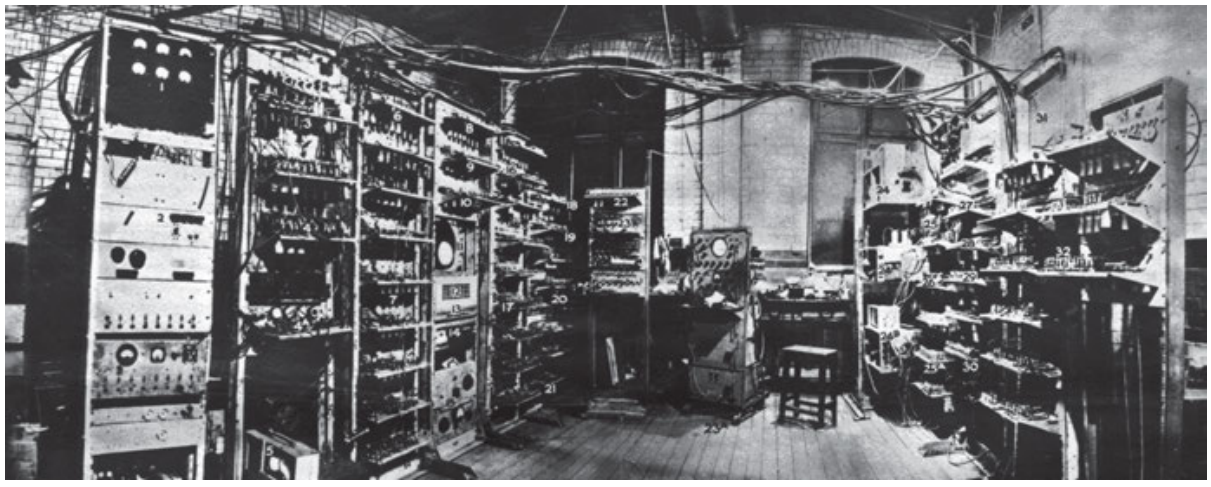

Abb. 2: 1949: MARK I, University of Manchester. Prototyp.

\section{Turing, Ashby und McCulloch - Ratio-Club und MACY-Konferenzen}

$\left(\right.$ Etwa $\left.^{57}\right)$ am 19. November 1946 antwortet Turing auf eine Anfrage Ross Ashbys, ob der von der Royal Society am National Physics Laboratory unter Leitung von Max Newmann in Bau befindliche Großrechner ACE - die Automatic Computing Engine - in der Lage sei, Nerventätigkeit zu simulieren. Diese Anfrage hat einen sehr weitreichenden Hintergrund, denn Ashby wollte ein bahnbrechendes experimentelles Gerät nicht materiell löten, sondern als Programm funktional virtualisieren. Dieses spezielle Gerät sollte die Selbststeuerung eines Systems kommunizierender Einzelartefakte verkörpern und zu einem Leitartefakt - einem epistemischen Ding - der frühen britischen Kybernetik werden: Der Homeostat hätte nicht analog mit Schwingspulen gebaut, sondern als Programm entworfen werden können.

Die Anfrage wurde von Sir Charles Darwin - dem Direktor der Royal Society weitergegeben. Turing antwortet:

In working on the ACE I am more interested in the possibility of producing models of the action of the brain than in the practical applications to computing. [...]. The ACE will be used, as you suggest, in the first instance in an entirely disciplined manner, similar to the action of the lower centres, although the reflexes will be extremely complicated. The disciplined action carries with it the disagreeable feature, which you mentioned, that it will be entirely uncritical when anything goes wrong. It will also be necessarily devoid of anything that could be called originality. There is, however, no reason why the machine should always be used in such a manner: there is nothing in its construction which obliges us to do so. It would be quite possible for the machine to try out variations of behaviour and accept or reject them in the manner you describe and I have been hoping to make the machine do this. This is possible because, without altering the design of the machine itself, it can, in theory at any rate, be used as a model of any other machine, by making it remember a suitable set of instructions. The ACE is in fact analogous to the ,universal machine' described in my paper on computable numbers. [...] Thus, although the brain may in

57 So handschriftlich ergänzt auf Turing 1946 (Add MS 89153/26). 


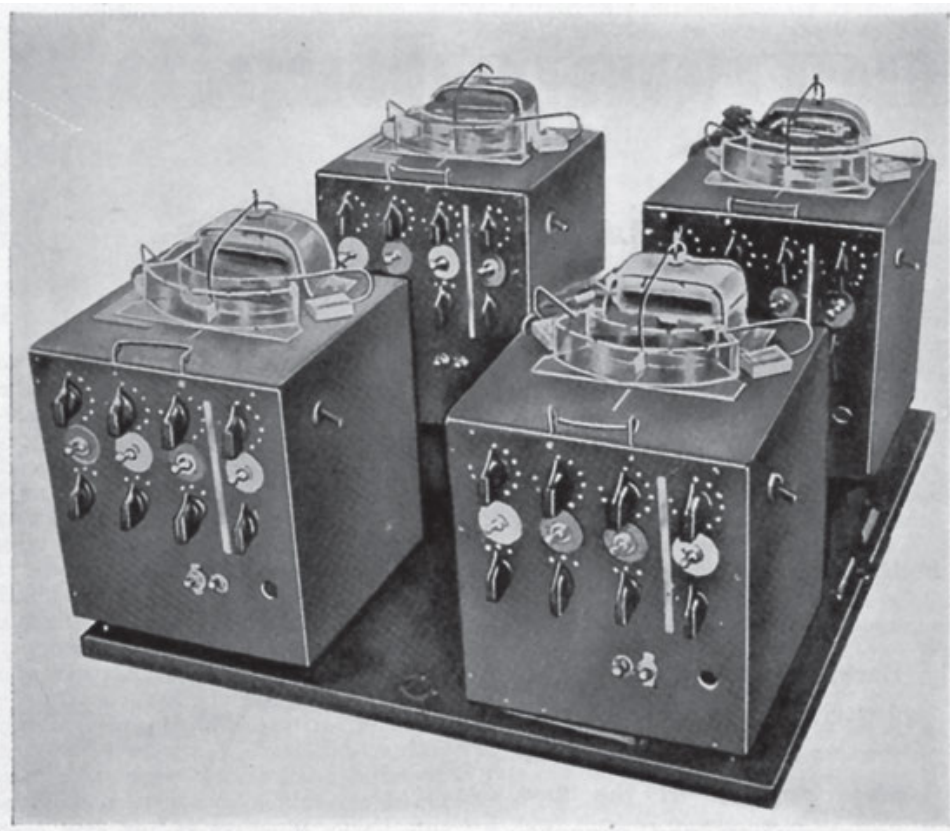

Fig. 1-The homeostat, with its four units, each one of which reacts on all the others.
Abb. 3:

1949: Ashbys Homeostat.

fact operate by changing its neuron circuits by the growth of axons and dendrites, we could nevertheless make a model, within the ACE, in which this possibility was allowed for, but in which the actual construction of the ACE did not alter, but only the remembered data, describing the mode of behaviour applicable at any time. ${ }^{58}$

Ashby nahm Turings Angebot nicht an, das Modell wurde gebaut, nicht simuliert. Seine Gründe hierfür sind der Forschung - bisher - unbekannt.

Es blieb allerdings nicht bei einem kurzen Briefwechsel zwischen Ashby und Turing. Von 1949 bis $1955^{59}$ traf sich der britische Ratio-Club in London - eine gelehrte Gesellschaft zur Erforschung der Folgen der Veröffentlichungen Norbert Wieners durch solche gelehrten Mitglieder, die „Kybernetik schon gedacht hätten, bevor Wieners Buch herauskam“. ${ }^{60}$ Neben Turing und Ashby war auch Jack Good Mitglied, der ehemalige Kriegsmitarbeiter Turings, der später den Begriff der ,Superintelligenz entwickelt hat. Am 7. Dezember 1950 sprach Turing dort über Educating a Digital Computer - zumindest finden sich Termin und Titel in den überlieferten Aufzeichnungen. Im Gegensatz zu den MACY-Konferenzen in den Vereinigten Staaten wurde das Vorgetragene nicht gesammelt in den Druck gegeben.

58 Turing 1946 (Add MS 89153/26).

59 Mit einem Abschlusstreffen 1958 im Anschluss an das „Mechanisation of Thought Prozesses“Symposium am National Physical Laboratory.

60 Husbands/Holland 2008, 101 [meine Übersetzung]. 


\section{US-Kybernetik und die MACY-Konferenzen}

Auch die US-Amerikanischen Kybernetiker bemühten sich um Turing. Warren McCullogh, mit Walter Pitts der Urheber des mathematisierten Modells der Nervenübertragung ${ }^{61}$ (eines neuen Feedback-Calculus), war auch Hauptorganisator der MACY-Konferenzen, die in den USA die maßgeblichen Nachkriegs(computer)forscher zusammenbringen sollten, um die Ähnlichkeit der Rechenmaschinen zum menschlichen Gehirn zu erforschen. Diese jährlichen Konferenzen sollten zur Sammelstelle

Diagrammatic Symbol

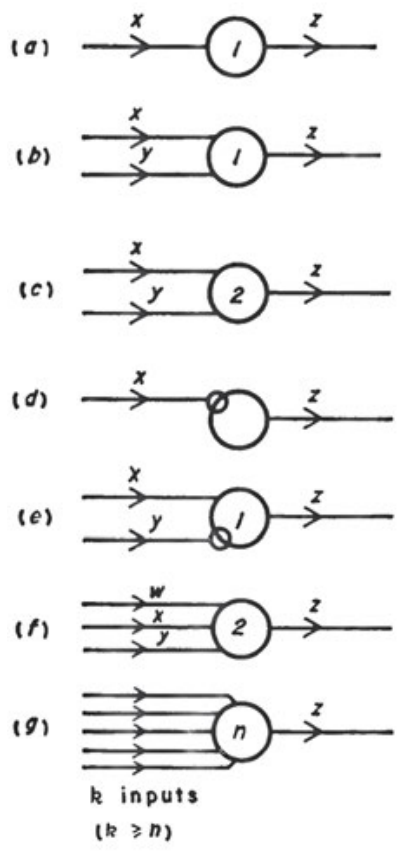

Characteristic

Excitation

Excitation, "or"

Excitation, "and"

Inhibition (overrides any excitation)

\section{Excitation} (threshold 2)

Excitation (threshold $n$ )
Operation

Signal out (z) if sig- $z=x$ nal in $(x)$

Signal out (z) if sig- $z=x \vee y$ $\mathrm{nal}$ in on either line $\quad(x$ or $y)$ $(x, y)$ (or both)

Signal out $(z)$ if sig- $z=x \& y$ nals in on both lines $\quad(x$ and $y)$ $(x y)$

Signal in $(x)$ inhibits signal out

Signal out $(z)$ if sig- $\quad z=x \&(\sim y)$ nal $x$ in and no sig- $\quad[x$ and (not $y)]$ nal $y$ in

Signal out $(z)$ if sig- $z=(w \& x) \vee(x \& y)$ nals in on two or $\quad \mathrm{V}(y \& w)$ more lines $(w, x, y)$

Signal out $(z)$ if signals in on $n$ or more lines

Delays:

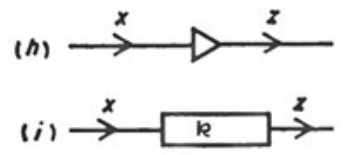

1 pulse-period delay $\quad z(t+1)=x(t)$, or $z_{1}=x_{0}$

$k$ pulse-periods de- $z(t+k)=x(t)$, lay or $z_{k}=x_{0}$

FIG. 53. Functional elements of a serial machine.

Abb. 4: 1949: Hartrees Diagrammatik der McCullogh/Pitts /von Neumann/Turing-Notation automatisierter Rechenprozesse.

61 McCullogh/Pitts 1943. Hier wird ausführlich Turing 1936 zitiert. 
der Kybernetiker in der westlichen Welt werden, ihre Ergebnisse zirkulierten. ${ }^{62} \mathrm{Zu}$ den geladenen Gästen zählte auch Alan Turing, der allerdings aus verschiedenen Gründen im Jahr 1953 absagte:

Dear McCullogh, it was very gratifying and tempting to get your invitation to the Macy meeting.
You have certainly got a wonderful collection of people together. If it were in Europe I should
certainly try to make it, but I am really rather a stay-at-home type. Unfortunately also it is during
term time, and I am doubtful if I could get permission to be away. Yours sincerely A. M. Turing. ${ }^{63}$

Abgesehen vom kulturhistorischen Eindruck, den diese Zeilen aus dem Kalten Krieg über die mit ihm verbundenen sicherheitsgetriebenen Repressionen für die Zivilbevölkerung vermitteln, ${ }^{64}$ belegt diese exklusive Einladung die Einschlägigkeit von Turings Forschung für die US-Kybernetik. McCullogh und Pitts richtungsweisende Veröffentlichung zum ,Calculus der Nervenzellen` zitiert schon 1943 Turings zentrale Überlegungen zur Universellen Rechenmaschine. Sie betonen hier ihre Überzeugung, dass auch hochgradig analoge Phänomene (wie das menschliche Nervensystem) auf Turings diskreten Maschinen berechnet werden können - oder sogar diskrete Turing-Maschinen sind. ${ }^{65}$ Können wir aber davon ausgehen, dass Turing selbst Feedbackgerät kannte und ernst nahm? Gibt es ,Ungenanntes‘, das diese Vermutung wahrscheinlich macht?

\section{4 - Blacks Box - der Bell-Telephonnetz-Feedback- Verstärker als zentrales Bauteil der Turing-Galaxis}

Wir können verschiedene Geschichten erzählen, wie es dazu gekommen ist, dass ,Schwarze Kästen ' ihre spezielle Metaphorik der ,dunklen Verschlossenheit‘ in unsere Gedankenfelder und Diskurse einspeisen. ${ }^{66}$ Diese Geschichten sind mitunter spannend und aufschlussreich; sie zeigen viel von Zeit und Kontext, für die sie erzählt wurden, oder leuchten die Vergangenheit auf jeweils unterschiedliche Art und Weise aus und machen so Geschichte. Ihr Stil verändert die Leserhaltung und Stimmmung: Im hohen, heroischen Ton lassen sich Geschichten selbstzerstörender Beutegeräte im Zweiten Weltkrieg erzählen, die nur unter großem persönlichen Risiko untersucht

\footnotetext{
62 Siehe Pias 2004.

63 Undatierter Brief, von Pias einsortiert zwischen dem 15. und dem 16. Januar 1953.

64 Turings mangelnde Reiseerlaubnis - so scheint es durch - ist durchaus nicht nur universitätsorganisatorischer Art. Auch etwas Ungenanntes spielt eine Rolle. Eine Überseereise war für einen Geheimnisträger des Zweiten Weltkriegs offensichtlich in den 1950er Jahren und nach einem unappetitlichen Gerichtsprozess wegen gelebter Homosexualität keine Selbstverständlichkeit.

65 McCullogh 1949, 492: „The brain is a logical machine.“

66 Einen Überblick liefert die Einleitung in diesem Band.
} 
werden konnten - den Verlust des unersetzlichen Gerätes ${ }^{67}$ oder eigener Gliedmaßen durch Explosionen immer im Blick. Im niederen, weltläufigen Ton kann man über die Farbgebungspraxis früher Flugschreiber oder der Transportkisten des Geheimmittelversandes im Zweiten Weltkrieg (Zeit-)Zeugnis ablegen und so betonen, wie auch abstrakte Konzepte durch Banalitäten benannt werden können. ${ }^{68}$ Manchmal gibt es aber überraschende Funde, die auf spannende Spuren verweisen: Einer dieser Funde ist ein technischer Aufsatz eines Elektroingenieurs der Zwischenkriegszeit. Dieser Spezialist für Nachrichtentechnik publizierte in den Mitteilungen der Bell Laboratories im Januar 1934 auf wenigen Seiten Prinzipien und Schaltkreise eines elektronischen Bauelements, das in der Steuerung von Telegraphen- und vor allem Telephonnetzen eingesetzt werden sollte - in jedem Datennetz, dessen Informationen als Modulation einer Trägerwelle kodiert werden. Hier hatte es dafür zu sorgen, dass Rauschen verringert und trotzdem Leistung verstärkt werden kann. Dieses Bauteil ist der Integrated Feedback Amplifier, sein Erfinder trägt den Namen Harold Stephen Black. Black entwarf einen Feedbackkasten zur automatisierten Selbsteinregelung von Nachrichtensignalen - ,Blacks Box‘ als Wortbildung im naiven Sinne. Dieses Schaltelement sollte ein lebensweltliches nachrichtentechnisches Problem lösen; es wurde als Bauteil in ein gegebenes Maschinenarrangement eingefügt und als Artefakt auf eine Einsatzpraxis hin gestaltet. Seine zentrale Operation ist das Feedback - nicht der Filter - und zwar über ,Anreicherung“ des Signals - nicht dessen ,Reinigung‘. Es geht um gegenseitiges Verstärken von gewünschten Signalen in einem Prozess der Selbst- und nicht der Fremdsteuerung. Wechselwirkung - schon bei Kant Merkmal des Organischen ${ }^{69}$ wird herbeigeführt und genutzt, um eine mechanische Abfolge von Regel-Befehlen zu ersetzen. Aus einer Wirkkette der effizienten Folge wird eine wechselwirkend schwingende Annäherung an ein finales, als Maschinenzweck vorgegebenes Ziel. Dass es hier darum geht, sequentielle symbolische Steuerung durch maschinelles Lernen $\mathrm{zu}$ ersetzen - und somit von Mechanischer Intelligenz zu autooperativen Mechaniken zu wechseln - konnte Black (in gewissem Sinne) noch nicht formulieren: Ihm fehlte das Konzept des ,Programms‘, das erst Alan Turing als formgebende Praxis über sein universelles Modell der Symbolverarbeitung legt, um rechnend-im-Raum

67 Ashby verwendet verschiedene Fallbeispiele, so auch die Landmine, Ashby 1956, 92: „In its most dramatic form it occurs when the investigation of a new type of enemy mine leads to an explosionwhich can be described more abstractly by saying that the system has passed from a state to which no manipulation at the input can make the system return.“

68 Eine gute, wenn auch leider unreferenzierte Zusammenstellung findet sich bei Ashby (1954) in seiner sehr lebendigen Vorstellung des Black Box-Konzeptes (Kap.6.1). Hier finden sich die Bombe, das Telefonnetzwerk oder die Labormaus-im-Labyrinth als Beispiele, aber auch das die Gegenstände seiner Spielwelt erkundende Kind - oder das unbekannte Objekt, das von einer fliegenden Untertasse fallen gelassen wurde. Winkler (2014) geht vom absturzsicheren Flugschreiber als Bildgeber aus und konzentriert sich auf die Handlungen der Black Box-Herstellung - dem Black Boxing (als Verb) am Beispiel des Zusammenfaltens eines Pappkartons.

$69 \mathrm{KdU}$ §65, 373f., hierbei dem ,Grundsatz der Gemeinschaft‘ (commercium) (KrV B 256) folgend. 
den rechnenden Raum zu erschließen. Was Black aber sehr bewusst war, ist die Auswirkung seiner Bauelemente auf die Signal-to-Noise-Ratio und somit auch auf die Produktionsprozesse von Schaltanlagen und Kommunikationsgerät. Seine Apparate, so schrieb er, seien konzipiert worden für Verwendung im Verbund mit den besten Bauteilen (wie zum Beispiel höchstwertigen Radioröhren) und könnten so exzellente Ergebnisse noch weiter verbessern. Gleichzeitig sei es aber auch möglich, minderwertige Bauteile im Verbund einzubetten - und mit Hilfe der Selbstverstärker-Regelkreise aus Mangelware brauchbares Material für die Nachrichtentechnik zu machen. Er setzte hiermit einen Impuls, der ein Jahrzehnt später in die Debatte um die geeigneten Bauteile von Großrechnern eingespeist werden sollte: John von Neumann wird sich fragen, wie zuverlässige Maschinen aus unzuverlässigem Material erbaut werden können ${ }^{70}$ - und zwar in der Absicht, mit den Höchstpreisröhren ein Nadelöhr der Rechenmaschinentechnik zu umgehen und diese in Krisenzeiten durch einfachste Radioröhren zu ersetzen.

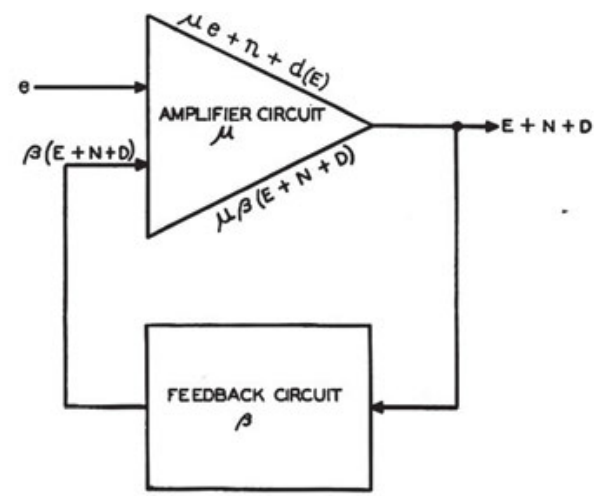

Fig. 1-Amplifier system with feedback.

$e$-Signal input voltage.

$\mu$-Propagation of amplifier circuit.

$\mu e$-Signal output voltage without feedback.

$n$-Noise output voltage without feedback.

$d(E)$-Distortion output voltage without feedback.

$\beta$-Propagation of feedback circuit.

E-Signal output voltage with feedback.

$\mathrm{N}$-Noise output voltage with feedback.

$D$-Distortion output voltage with feedback.

Abb.5: 1934: Blacks Box, Diagramm.

Festgehalten werden kann, dass Feedback als Prinzip und als Bauteil zu Turings Zeit unter spezialisierten Ingenieuren geläufig war und sich für Turings Fall zeigen lässt, dass seine Diskursnetzwerke ihn an eine besondere Form der Feedbackforschung ankoppelten. Mit Rückgriff auf die obigen Ausführungen zu den Grenzen der Brauchbarkeit der logischen Analyse zeigt sich dies deutlich durch Relektüre einer opaken, unzugänglichen und stark metaphorischen Passage seines Aufsatzes von 1950.

70 Von Neumann 1956. 


\section{Turings Versuche, den ,Einwand der Lady Lovelace“ mit überzeugenden Metaphern zu entkräften}

Man kann in einer wissenschaftlichen Publikation nur ,hart' am Argument bleiben, man kann aber auch ,weich' versuchen, zu überzeugen. ${ }^{71}$ Nicht nur zwingende Logik, sondern auch Stil und Schreibform - und vielleicht sogar unser Schreib- und Lesegerät $t^{72}$ - sind eingebunden in den Prozess der Entstehung, Bewertung und Weitergabe von Wissen. Dies formuliert auch Alan Turing 1950 in seinem zentralen Aufsatz Computing Machinery and Intelligence im Abschnitt zu den ersten und zukunftsweisenden Grundlagen Maschinellen Lernens:

These last two paragraphs do not claim to be convincing arguments. They should rather be described as "recitations tending to produce belief“ “ ${ }^{73}$

Sprachhandlungen - Rezitationen - müssen für Turing nicht notwendigerweise argumentativ und zwingend sein, um in einer wissenschaftlichen Publikation Platz zu haben. Dies gilt vor allem, wenn er versucht, seine eigene Haltung zu plausibilisieren, nachdem er den Stand der Forschung dargestellt und neun typische Gegenargumente in einer heuristischen Doxographie der Gegnerschaft entkräftet hat.

Das ,Argument der Lady Lovelace‘ („Eine Maschine könne nur ausführen, was man ihr gesagt habe“ $)^{74}$ hält Turing für ein starkes Gegenargument, auf das er auch in späteren Schriften wieder zurückkommen wird. Er schreibt 1950, er diskutiere es oft und widersprüchlich mit Kollegen, ${ }^{75}$ und bespricht es an zwei Stellen im Text.

Dieser ,Einwand der Lady Lovelace“ würde mit zwei Annahmen zusammenhängen: Rechenmaschinen würden nichts Neues hervorbringen können und das Verhalten von Rechenmaschinen könne einen kundigen Betrachter bei hinreichender Information nicht überraschen. Beide Annahmen verneint Turing unter Rückgriff auf den Diskurs seiner Zeit - an dieser Stelle sollen allerdings nicht seine ,harten' Argumente, sondern drei Metaphern ${ }^{76}$ interessieren, die er anführt, um die Leserschaft für seine Haltung einzunehmen.

71 Dass Turing selbst der zwingenden Kraft des Syllogismen nicht in jeder Situation traute, wurde bereits oben ausgeführt.

72 So zitiert Friedrich A. Kittler einen Brief Friedrich Nietzsches vom Februar 1882: „Unser Schreibzeug arbeitet mit an unseren Gedanken.“ (Kittler 1989, 293).

73 Turing 1950, 455.

74 Bei Lovelace: „The machine can do whatever we know how to order it to perform.“

75 Zum Beispiel namentlich genannt Hartree.

76 Turing 1950, 454f., Kap. „Learning Machines“. 


\section{Der menschliche Geist als Klavier}

Zunächst stellt er sich vor, dass ein Argument in eine Maschine „injiziert“ werden könne und beschreibt die Wirkung dieses Vorgangs, indem er diese Maschine mit einer Klaviermechanik vergleicht: ${ }^{77}$

Let us return for a moment to Lady Lovelace's objection, which stated that the machine can only do what we teil it to do. One could say that a man can, inject' an idea into the machine, and that it will respond to a certain extent and then drop into quiescence, like a piano string Struck by a hammer. $^{78}$

Hier muss - und dies steht nicht bei Turing - ein Detail des technischen Aufbaus des Pianos ergänzt werden: Klavierseiten werden im Regelfall vollständig gedämpft, da sonst das Klavier unkontrolliert auf seine Umwelt reagieren und gespenstische Klänge von sich geben würde. ${ }^{79}$ Nur dann, wenn eine Taste gedrückt und dadurch eine Seite angeschlagen wird, wird die Dämpfung gelöst - der Ton kann erklingen (wobei die Dauer des Nachklangs per Pedal geregelt werden kann).

Turing ruft so die akustische Kategorie der ,Resonanz' auf - in diesem Beispiel noch im Normalfall technisch gedämpft, das Signal verklingt nach einiger Zeit.

Bemerkenswert an dieser Stelle ist auch der Wechsel von einer Semantik der Zeit in eine Semantik des Raumes: Nicht mehr eine potentiell endlose Folge abgegrenzter Symbole, die regelgeleitet auf einem endlosen, aber binnendifferenzierten, diskret geteilten Schreib-/Leseband manipuliert werden, ist Objekt der Rechenoperationen, sondern analoge Wellen - überlappungsfähig, schichtbar und additiv.

\section{Der super-kritische Geist}

Dieser Metapher der ,im Regelfall gedämpften Resonanz‘ stellt Turing zwei eigene Bilder entgegen. Das erste entstammt der Atomphysik - ein mechanischer Geist könne „sub-kritisch“ sein, aber durchaus „super-kritisch“ werden, wie eben der menschliche Geist auch:

Another simile would be an atomic pile of less than critical size: an injected idea is to correspond to a neutron entering the pile from without. Each such neutron will cause a certain disturbance which eventually dies away. If, however, the size of the pile is sufficiently increased, the distur-

77 Oliver Schlaudt gab mir den Hinweis, dem Geist-Klavier-Vergleich in die Französische Aufklärung nachzuspüren. Dies soll an anderer Stelle geschehen.

78 Turing 1950, 454.

79 Dank für Hinweise und Anschauungsobjekt an Wolfgang Schröter, dessen „Enrico Caruso Grand“ während einer Generalüberholung (und Wartung der eingekoppelten MIDI-Mechaniken) auseinander- und wieder zusammengesetzt wurde. 
bance caused by such an incoming neutron will very likely go on and on increasing until the whole pile is destroyed. Is there a corresponding phenomenon for minds, and is there one for machines? ${ }^{80}$

Diese Metapher baut er aus und fragt sich, ob es auch menschliche Geister gäbe, die „sub-kritisch“ arbeiten würden - die also, wenn eine Idee in sie hineingefüttert wird, weniger als eine Idee wieder ausgeben:

There does seem to be one for the human mind. The majority of them seem to be sub-critical, i. e. to correspond in this analogy to piles of subcritical size. An idea presented to such a mind will on average give rise to less than one idea in reply. A smallish proportion are super-critical. An idea presented to such a mind may give rise to a whole theory consisting of secondary, tertiary and more remote ideas. Animals minds seem to be very definitely sub-critical. Adhering to this analogy we ask, „Can a machine be made to be super-critical?“. ${ }^{81}$

Bleibt man im Bild, schlägt Turing also vor, von einem Regelbetrieb der gedämpften Resonanz - wie in der Babbage/Lovelace'schen Mechanik - in einen Regelbetrieb der ungedämpften Resonanz überzugehen: der ,Turing Maschine 2.0` (oder: f-typemachine). Dann wären Effekte möglich, wie wir sie von „super-kritischen“ Geistern kennen. ${ }^{82}$

\section{Der Geist als Zwiebel}

Eine dritte Metapher, die Turing einführt, ist die des ,Häutens der Zwiebel'. In Betrachtung des menschlichen Geistes, so Turing, ließen sich Operationen finden, die vollständig mechanisch erklärt werden könnten. Wir würden jedoch dazu neigen, diese mechanisch erklärbaren Operationen nicht mehr zum Geist zu zählen, sobald sie erklärbar seien - wir würden sie als Haut von unserer Geist-Modell-Zwiebel abziehen. Dies würden wir Schicht-für-Schicht durchführen können, in der Hoffnung, unter der nächsten Haut den „echten Geist“ in der Maschine zu finden:

The ,skin of an onion' analogy is also helpful. in considering the functions of the mind or the brain we find certain Operations which we can explain in purely mechanical terms. This we say does not correspond to the real mind: it is a sort of skin which we must strip off if we are to find the real mind. But then in what remains we find a further skin to be stripped off, and so on. ${ }^{83}$

80 Turing 1950, 454.

81 Turing 1950, 454.

82 Dies hätte auch Folgen für unsere Theorie des Geistes und somit für unser Menschenbild.

83 Turing 1950, 454. 
Für Turing steht die Erfolgsaussicht dieses Verfahrens jedoch in Frage. Es sei möglich, so Turing, dass es eine letzte Zwiebelschale gäbe und unter dieser Schale nichts Weiteres mehr zu finden sei. Das Verfahren des Häutens der Zwiebel habe also eine Abbruchbedingung - es ist keine Haut mehr da:

Proceeding in this way do we ever come to the real' mind, or do we eventually come to the skin which has nothing in it? In the latter case the whole mind is mechanical. (It would not be a discrete-state machine however. We have discussed this. $)^{84}$

Die in diesen Metaphern eingesiegelten Konzepte sollten in den Folgejahren historisch wirksam werden. Zum einen wird in die Erzählung vom ,Häuten der Zwiebel‘ bei Turing die Möglichkeit einer ,letzten leeren Haut' eingeschrieben und so eine vollständig stoffgebundene Theorie des Geistes vorbereitet, die in einer material-reduziblen Radikalität auf Nicht-Stoffliches verzichtet. Zum anderen ist bei Abwesenheit einer nicht-stofflichen Komponente die (geeignete) Maschine aus dieser Sicht ein Kandidat für ,Intelligenz - aus der Suche nach einem „super-kritischen“ mechanischen Geist wird die Queste nach einer „Technologischen Singularität“85.

\section{The stars can't be rushed}

Im gegenwärtigen kognitiven Raum wird oft hinweggewischt über ,technologische Singularität‘, ,Omega-Punkte‘, ,Uploading‘ und andere Bewältigungsstrategien des Umgangs mit der „beschleunigten Beschleunigung“"86 der Entwicklung aller Lebensbereiche. Dies gibt Anlass für die digitalen Geist(er)beschwörungen des Post- und Trans-Humanismus.

Begibt man sich jedoch auf die Suche nach den Entstehungsbedingungen ${ }^{87}$ dieser gegenwärtigen Erzählungen, finden sich schon im zentralen Artikel von Alan Turing 1950 Spuren, die in die Geschichte der Arbeitsorganisation führen.

,Künstliche Intelligenz - als gegeben vorausgesetzt - soll in der Gegenwart als Artefakt die Basis der ,Superintelligenz‘ bilden. Doch dieses ,Arte-Fakt‘ ist ein Sprachspiel, das sich als ,Kunst-Produkt‘ verselbständigen konnte. Diese Erzählungen wirken als ,Mem` - und nicht in rem. Aber nicht nur die agency eines Elektronenhirns

84 Turing 1950, 455.

85 Wie dies Irving John ,Jack“ Good 1965 vorbereitet: als ehemaliger Schüler und Mitarbeiter Turings an der Government Code and Cypher School während des Zweiten Weltkriegs verwendet er turing'sches Vokabular, prognostiziert aber eine „intelligence explosion“ des Computers (Good 1965, 33). Er beriet außerdem Stanley Kubrick während der Entwicklung und der Dreharbeiten von 2001. Der Ausdruck „technologische Singularität“ zuerst bei Vinge 1993.

86 Toffler 1970, passim.

87 Gleichermaßen im Sinne von Kittlers „historischem a priori“ und Foucaults „Dispositiv“. 
oder robotic companions oder Cyborg-Phantasien stehen als narrative Zukunftsbeschwörung im Debattenraum der Gegenwart. Auch die Handhabbarkeit und Affordanz der allgegenwärtigen, unsichtbaren, vernetzten Rechenmaschinen müssen geklärt werden; Geschichten ihrer historischen Genese bieten uns Orientierungswissen in der Gegenwart. Die ,Künstliche Intelligenz des Weltkrieges ${ }^{68}$ als Automatisierung der Kopfarbeit ${ }^{89}$ ist die Spur, die ich vorschlage, aufzunehmen..$^{90}$

\section{Gegenwärtige Wissenschaftstheorie im Vokabular der Computerzeit}

In der Gegenwart verwenden einige wissenschaftliche Disziplinen Vokabular, das aus den historischen Computerwissenschaften entlehnt ist. Turing galt schon zu seiner Zeit als wesentlicher Urheber der Kulturtechnik des Programmierens, des Erstellens eines strukturierten Schrittfolgeplans, ${ }^{91}$ weshalb die Spur des Programmier-Begriffs bei Turing aufgenommen werden kann. ${ }^{92}$ Diese Spur läuft über die Akteur-NetzwerkTheorie Bruno Latours, ${ }^{93}$ die prominent den Programm-Begriff verwendet und so in den theoretischen Wortschatz der Sozial- und Geisteswissenschaften hineinkopiert. Im gleichen theoretischen Vokabular findet sich bei Latour auch die ,Black Box‘, die als Konzept mindestens die Science and Technology Studies und ihre Anschlussdisziplinen seit den 1980er Jahren beschäftigt. Auch wenn Turing selbst an keiner Stelle „Black Box“ schreibt, kann in diesem Beitrag nachgewiesen werden, dass er ebenfalls über das Konzept, wenn nicht das Wort, der technischen Black Box - als FeedbackApparat - verfügte.

88 Um Kittler 1990 aufzugreifen.

89 Bisher als Dokumentation: Vater/Bertolo 2019.

90 Ich hätte sie nicht gefunden ohne den Versuch, Black Boxes zu öffnen, Handlungsprogramme zu hinterfragen und Anti-Programme einzuüben. Eine Dispositivanalyse vorzunehmen ist vielleicht so etwas, wie ein Anti-Programm abzuarbeiten.

91 Hartree 1949, 102.

92 Zur Zeit der Fertigung dieses Beitrags ist dem Verfasser keine ältere Verwendung von ,programmieren` vor Turing bekannt (auch nicht in Wexelblat 1981 zur Geschichte der Programmiersprachen; Russell/Norvig 2010 [1995] diskutieren die Wortherkunft nicht, sondern verwenden ,programming language، unhinterfragt und undefiniert als Teil ihrer Normalsprache). Zur Frage, ob to program synonym mit to code verwendet wurde, schon Hartree 1949, 54, dort mit beginnender Differenzierung als Bezeichnungen für Abschnitte in einem Prozessphasenmodell (,Programm' als strukturierter Entwurf, ,Code' als Übersetzung in eine maschinenlesbare formale Sprache). So und deutlich auch Hopper 1955, 1, mit den Arbeitsphasen: „Analysis, Programming, Coding, Debugging, Production, Running, Evaluation“ - ,Program“ als Plan, ,Code‘ als (technische) Ausführung. Bei Goldstine/von Neumann 1947, 24 folgende noch unscharfe Formulierung: „Before we start on this program of coding specific problems“, die allerdings auf eine Hierarchie von ,Programm“ und ,Code‘ deutet.

93 Latour/Akrich 1992, erweitert Latour 1999, $303 \mathrm{ff}$. 
Die ,Black Box Künstliche Intelligenz löste jedenfalls in der Gegenwart Irritationen aus, die als Stör-Signale in die Feedbackschleifen unseres komplexen rückkopplungsgesteuerten diskursiven Systems ,Philosophie‘ eingestreut wurden. Diese Irritation gilt es zu lösen - auch durch genaues Lesen in einer gut ausgestatteten Bibliothek und Entschleunigung des Denkens. Zwar gibt es prinzipiell keine Einwände gegen die Möglichkeit intelligenter Maschinen, ${ }^{94}$ doch lässt sich festhalten, dass sich eine Künstliche Intelligenz in unsere Welt noch nicht manifestiert hat. Ihre Entwicklung steckt, wenn überhaupt, noch im „Kambrium“, wie es Oswald Wiener formulierte. ${ }^{95}$

Von Singularitäten sollten wir uns keine Angst machen lassen. Um mit Steward Brand, Chronist der Westküsten-Aussteiger-Utopie, Begleiter der Entstehung des Silicon Valley, Baumeister der ,Uhr der langen Gegenwart‘ und Verleger des ersten Fotos der „Whole Earth“ zu enden - auch das Empfinden der beschleunigten Beschleunigung geht vorbei: „The stars can’t be rushed. Whew, that's a relief.“96

\section{Literaturverzeichnis}

Ashby, W. Ross (1949), „The Electronic Brain“, in: Radio-Electronics March 1949, 73-80.

Ashby, W. Ross (1956), An Introduction to Cybernetics, London.

Babbage, Charles (1864), Passages from the Life of a Philosopher, London.

Beauclair, Wilfried de (1968), Rechnen mit Maschinen. Eine Bildgeschichte der Rechentechnik, Braunschweig.

Becker, Rainer C. (2012), Black Box Computer. Zur Wissensgeschichte einer universellen kybernetischen Maschine, Bielefeld.

Black, Harold Stephen (1934), „Stabilized feedback amplifiers“, in: The Bell System Technical Journal (Januar 1934), 1-18 (auch in: Electrical Engeneering (Januar 1934), 114-120).

Bolter, David J. (1984a), Turings' Man. Western Culture in the Computer Age, Chapel Hill.

Bolter, David J. (1984b), „Artificial Intelligence“, in: Daedalus 113 (3), 1-18.

Bolter, David J. (1997), „Das Internet in der Geschichte des Schreibens“, in: Stefan Münker/Alexander Roesler (Hgg.), Mythos Internet, Frankfurt a. M., 37-55.

Brand, Steward (1977), „The Long View“, in: Space Colonies (CoEvolution September 1977), https:// space.nss.org/settlement/nasa/CoEvolutionBook (Stand: 19.9.2019).

Churchill, Sir Winston S. (1949), The Second World War. Bd. 2: Their Finest Hour, London.

Cooper, S. Barry/Hodges, Andrew (Hgg.) (2016), The Once and future Turing. Computing the World, Cambridge.

Cooper, S. Barry/van Leeuwen, Jan (Hgg.) (2013), Alan Turing. His Work and Impact, Amsterdam.

Copeland, Jack B./Bowen, Jonathan P./Sprevak, Mark/Wilson, Robin/and others (2017), The Turing Guide, Oxford.

Coy, Wolfgang (1984), „METH-EMETH. Abenteuer der künstlichen Intelligenz“, in: Kursbuch 75 (,Computerkultur“), 1-11.

94 Lesenswert hierzu Schütt 2020 in diesem Band.

95 Wiener 1990.

96 Brand 1977. 
Davis, Martin (2000), The Universal Computer. The Road from Leibniz to Turing (Engines of Logic. Mathematicians and the Origin of the Computer), New York.

Dawkins, Richard (2006 [1976]), The Selfish Gene, Oxford.

Dotzler, Bernhard J. (2006), Diskurs und Medium. Zur Archäologie der Computerkultur, München.

Epstein, Robert/Roberts, Gary/Beber, Grace (Hgg.) (2007), Parsing the Turing Test. Philosophical and Methodological Issues in the Quest for the Thinking Computer, [ohne Ort].

Feynman, Richard (1985), Surely Your're joking, Mr. Feynman!, New York.

Foucault, Michel (2003 [1977]), „Das Spiel des Michel Foucault“, in: Schriften in vier Bänden. Bd. 3 (1976-1979), hg. von Daniel Defert u. François Ewald., aus d. Französischen von Michael Bischoff et al., Frankfurt a. M., 391-429. - folgt: ders. (1994), Dits et ecrits, Paris. - Erst.: ders. (1977), Le jeu de Michel Foucault, in: Ornicar? Bulletin periodique du champ freudien 10 (1977), 62-93.

Goldstine, Herman H. (1972), The Computer from Pascal to von Neumann, Princeton.

Goldstine, Herman H./von Neumann, John (1947), „Planning and Coding of Problems for an Electronic Computing Instrument", Report on the Mathematical and Logical aspects of an Electronic Computing Instrument 2 (1-3) (=IAS EXP 1946-57 No.4,8,11), Princeton.

Good, Irwing John (1965), „Speculations Concerning the First Ultraintelligent Machine“, in: Advances in Computers 6, 31-88.

Hartree, Douglas (1949), Calculating Instruments and Machines, Urbana.

Herken, Rolf (Hg.) (1995 [1988]), The Universal Turing Machine A Half-Century Survey (Computerkultur 2), Wien.

Hiller, Moritz (2019), „Computing. Zur Einführung“, in: Andreas Ziemann (Hg.), Grundlagentexte der Medienkultur. Ein Reader, Weimar, 163-168.

Hodges, Andrew (2014 [1983]), Alan Turing. The Enigma, London.

Hopper, Grace Murray (1955), „Automatic Coding for Digital Computers“, Remington Rand White Paper, http://www.bitsavers.org/pdf/univac/HopperAutoCodingPaper_1955.pdf (Stand: 19.12.2019).

Husbands, Philip/Holland, Owen (2008), „The Ratio Club. A Hub of British Cybernetics“, in: Philip Husbands, Owen Holland u. Michael Wheeler (Hgg.), The Mechanical Mind in History, Cambridge (MA), 91-148.

Ince, Darrel C. (Hg.) (1992), Mechanical Intelligence (Collected Works of Alan Turing 1), Amsterdam. Kant, Immanuel (1781A/1787B), Kritik der reinen Vernunft (Akademie-Ausgabe 3+4), Riga. Online: Bonner Kant-Korpus, korpora.zim.uni-duisburg-essen.de/kant/ (Stand: 19.9.2019).

Kant, Immanuel (1790), Kritik der Urteilskraft (Akademie-Ausgabe 5), Riga. Online: Bonner Kant-Korpus, korpora.zim.uni-duisburg-essen.de/kant/ (Stand: 19.9.2019).

Kittler, Friedrich A. (1984), Grammophon - Film - Typewriter, Berlin.

Kittler, Friedrich A. (1990), „Die Künstliche Intelligenz des Weltkriegs: Alan Turing“, in: Gerhhard Bur, Friedrich A. Kittler u. Horst Turk (Hgg.), Das Subjekt der Dichtung. Festschrift für Gerhard Kaiser, Würzburg, 187-202.

Kittler, Friedrich A./Dotzler, Bernhard J. (1987), „Alan M. Turing. Nachwort“, in: Friedrich A. Kittler u. Bernhard J. Dotzler (Hgg.), Alan M. Turing. Intelligence Service. Schriften, Berlin, 211-233.

Latour, Bruno (1987), Science in Action. How to follow scientists and engineers through society, Cambridge (MA).

Latour, Bruno (1999), Pandora's Hope. Essays on the Reality of Science Studies, Cambridge (MA), $303 \mathrm{ff} .$, Glossar.

Latour, Bruno/Akrich, Madeleine (1992), „A Summary of a Convenient Vocabulary for the Semiotics of Human and Nonhuman Assemblies“, in: Wiebe E. Bijker u. John Law (Hgg.), Shaping Technology / Building Society. Studies in Sociotechnical Change. Cambridge (MA), 259-265.

Laurel, Brenda (1991), Computers as Theatre, Boston.

Lavington, Simon H. (1975), A history of Manchester computers, Manchester. 
McCulloch, Warren S./Pitts, Walter H. (1943), „A Logical Calculus of the Ideas Immanent in Nervous Activity“, in: Bulletin of Mathematical Biophysics 5, 115-133.

McCulloch, Warren S./Pfeiffer, John (1949), „Of Digital Computers Called Brains“, The Scientific Monthly 69 (6), 368-376.

Metropolis, Nicholas/Howlett, Jack/Rota, Gian-Carlo (Hgg.) (1980), A History of Computing in the Twentieth Century. A collection of essays with introductory essay and indexes, Orlando.

Neumann, John von (1954), „Probabilistic Logics and the Synthesis of Reliable Organisms from Unreliable Components“, in: Claude Shannon u. John McCarthy (Hgg.), Automata Studies, Princeton.

Pias, Claus (2004), „Zeit der Kybernetik. Eine Einstimmung“, in: Claus Pias (Hgg.), Cybernetics. The Macy-Conferences 1946-1953. Bd.2, Essays and Documents, Zürich, 9-42.

Pratt, Vernon (1987), Thinking Machines. The Evolution of Artificial Intelligence, Oxford.

Quine, Willard Van Orman (1951), „Main Trends in Recent Philosophy: Two Dogmas of Empiricism“, in: Philosophical Review 60 (1), 20-43.

Riskin, Jessica (2016), The restless clock. A history of the centuries-long argument over what makes living things tick, Chicago/London.

Russell, Stuart J./Norvig, Peter (2010 [1995]), Artificial Intelligence. A Modern Approach, Upper Saddle River.

Schmeh, Klaus (2013), „Why Turing cracked the Enigma and the Germans did not“, in: S. Barry Cooper u. Jan van Leeuwen (Hgg.), Alan Turing. His Work and Impact, Amsterdam, 432-437.

Schütt, Hans-Peter (2020), ,,Du lebloses, verdammtes Automat!‘. Unser Schicksal vis-à-vis Maschinen und Automaten ...", in diesem Band.

Shieber, Stuart M. (Hg.) (2004), The Turing Test. Verbal Behavior as the Hallmark of Intelligence, Cambridge (MA).

Toffler, Alvin (1970), Future Shock, New York.

Turing, Alan M. (Nachlass King's College, Cambrige), GB 272 PP/AMT, www.turingarchive.org (Stand: 19.9.2019).

Turing, Alan M. (Nachlass Manchester), GB 133 TUR/Add., archiveshub.jisc.ac.uk/data/gb133-tur/ add (Stand: 19.9.2019).

Turing, Alan M. (1936), „On Computable Numbers, with an Application to the Entscheidungsproblem“, in: Proceedings of the London Mathematical Society 42, 230-265.

Turing, Alan M. (1946), Brief von Turing an Ashby, vermutlich 19. November 1946, GB 272 PP/AMT/ Add MS 89153/26, https://www.bl.uk/collection-items/letter-from-alan-turing-to-w-ross-ashby (Stand: 28.12.2019).

Turing, Alan M. (1950), „Computing Machinery and Intelligence“, in: Mind 59 (236), 433-460.

Vater, Christian/Bertolo, Christoph (2019), „300 Jahre Mechanische Intelligenz“, Dokumentarfilm, kim.hfg-karlsruhe.de/300-jahre-mechanische-intelligenz/ (Stand: 25.9.2019).

Vinge, Vernor (1993), „The Coming Technological Singularity. How to Survive in the Post-Human Era“, in: Vision-21. Interdisciplinary Science and Engineering in the Era of Cyberspace (NASA Conference Publication 10129), Cleveland, 11-22.

Wexelblat, Richard L. (Hg.) (1981), The History of Programming Languages, New York.

Wiener, Norbert (1961 [1948]), Cybernetics. Or control and communication in the animal and the machine, zweite überarb. Auflage, Cambridge (MA).

Wiener, Oswald (1990), „Kambrium der Künstlichen Intelligenz. Nachwort“, in: Herbert A. Simon: Die Wissenschaften vom Künstlichen (Computerkultur 3), übers. von Oswald Wiener, Berlin, 175-228.

Williams, Michael R. (1985), A History of Computing Technology, Englewood Cliffs.

Winkler, Hartmut (2014), „Black Box und Black Boxing - Zur Einführung“ (Manuskript Graduiertenkolleg Automatismen Universität Paderborn 14.10.14), homepages.uni-paderborn.de/winkler/ gk-black.pdf (Stand: 19.9.2019).

Zuse, Konrad (2007 [1970]), Der Computer - mein Lebenswerk, Heidelberg. 


\section{Bildnachweise}

Abb.1: Hartee 1949, 57.

Abb. 2: Lavington 1975, 7.

Abb. 3: Ashby 1949, 78.

Abb. 4: Hartree 1949, 98.

Abb. 5: Black 1939, 3. 